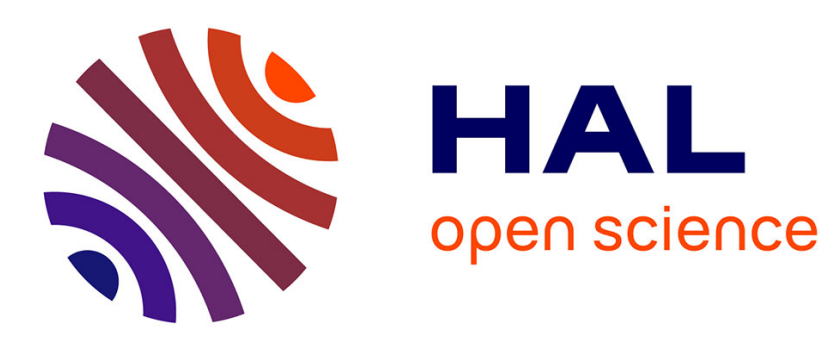

\title{
USE OF SUPERCONDUCTING MAGNETS IN MAGNETIC SEPARATION
}

\author{
M. Parker
}

\section{To cite this version:}

M. Parker. USE OF SUPERCONDUCTING MAGNETS IN MAGNETIC SEPARATION. Journal de Physique Colloques, 1984, 45 (C1), pp.C1-753-C1-758. 10.1051/jphyscol:19841153 . jpa-00223626

\section{HAL Id: jpa-00223626 https://hal.science/jpa-00223626}

Submitted on 1 Jan 1984

HAL is a multi-disciplinary open access archive for the deposit and dissemination of scientific research documents, whether they are published or not. The documents may come from teaching and research institutions in France or abroad, or from public or private research centers.
L'archive ouverte pluridisciplinaire HAL, est destinée au dépôt et à la diffusion de documents scientifiques de niveau recherche, publiés ou non, émanant des établissements d'enseignement et de recherche français ou étrangers, des laboratoires publics ou privés. 


\title{
USE OF SUPERCONDUCTING MAGNETS IN MAGNETIC SEPARATION
}

\author{
M.R. Parker \\ Department of Physicis, University of Salford, U.K.
}

Résumé - Un aperçu des possibilités de l'utilisation des systẽmes d'aimants supraconducteurs à champs forts pour la séparation magnétique sera donné. Une attention particulière est accordée aux avantages de la méthode de la boîte à mouvement alternatif pour la sëparation magnétique dans des champs à gradients forts, et à l'usage d'aimants quadrupolaires pour la séparation magnétique en gradients ouverts.

Abstract - A review is given assessing the potential for superconducting high-field magnet systems in magnetic separation. Particular attention is given to the advantages of the reciprocating canister approach to high gradient magnetic separation and to the use of quadrupole magnets in open gradient magnetic separation.

\section{I - INTRODUCTION}

Since about 1970 an increasing and widespread interest has developed in the design and working principles and in the commercial development of high field magnetic separation. This is commonly referred to $/ 1 /$ as 'high gradient magnetic separation' (HGMS). Much of the following is concerned with the development potential for superconducting (S/C) magnets in that technology. Other novel $\mathrm{S} / \mathrm{C}$ magnet systems used in magnetic separation are also reviewed briefly.

A crucial factor in any magnetic separation device is the magnetic traction force acting upon particles in the separating zone. For many of these though not a $11 / 2 /$ this force, $F_{H}$, is a magnetic dipolar interaction of the approximate form $/ 3 /$

$$
F_{M}=x V_{p}\left(B_{0} \nabla B_{0} / \mu_{0}\right)=x V_{p} f_{m}
$$

where $\nabla B_{0}$ is the spatial gradient of an external induction field, $B_{0}$, at the location of a particle of volume $V_{p}$ and of magnetic volume susceptibility $x_{p}{ }^{0}$ in a medium of corresponding value $x_{m}\left(x=x_{p}-x_{m}\right)$. In Eq. (1) $x$ and $v_{p}$ are exclusively properties of the particle and fluid medium. The quantity $f_{m}-$ a measure of the force density on the particles /4,5/ can be optimised by maximising $B_{0}$ and $\nabla B_{0}$ (the magnetic field gradient) independently over spatial dimensions appropriate to the particles. In HGMS, the gradient in $\mathrm{B}_{\mathrm{O}}$ is achieved by the insertion in the separating zone of a matrix of ferromagnetic fibres (or expanded metal screens). Above a threshold value of $B_{0}$ the majority of these fibres, wires, grids, etc., are magnetised to saturation in a direction transverse to their axes, whereupon $B_{0}$ approaches a limiting value of order $M_{S} / a / 2,5 /$ where $M_{S}$ is the saturation magnetisation of the fibres and $a$ is their (effective) radius of curvature. In HGMS systems, $f_{m}$ is, typically, of order $10^{11} \mathrm{Nm}^{-3}$ and increases linearly with increasing values of $B_{0}$. In HGMS, the large values of $f_{m}$ extend only over limited volumes of space adjacent to fibre surfaces in the separating zone and have a force range, therefore, of order a ( $50.1 \mathrm{~mm}$ ).

Other high-field separation devices are characterised by much smaller values of $f_{m}$ (of order $10^{8} \mathrm{Nm}^{-3}$ ) but by very much larger (0.05 to $0.1 \mathrm{~m}$ ) force ranges. Such devices, which achieve separation by particle deflection rather than by magnetic 


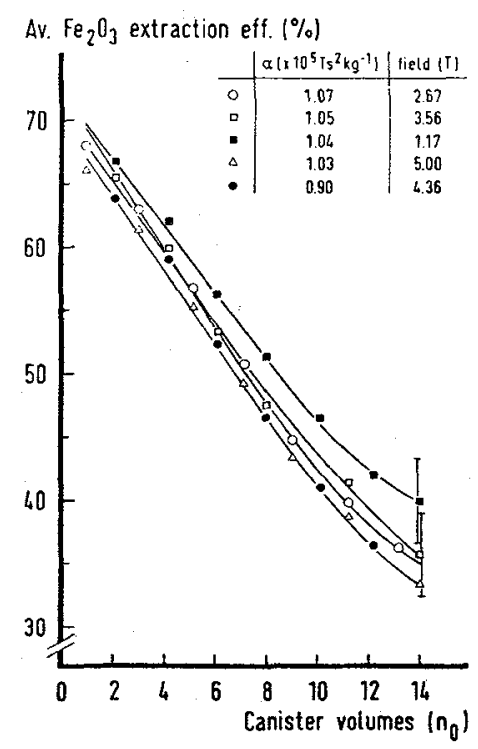

Fig. 1 - $\mathrm{Fe}_{2} \mathrm{O}_{3}$ recovery at various values of $\alpha$ (i.e. $\left.v_{m} / v_{0}\right)$. (Courtesy of J.H.P. Watson $77 / 9$

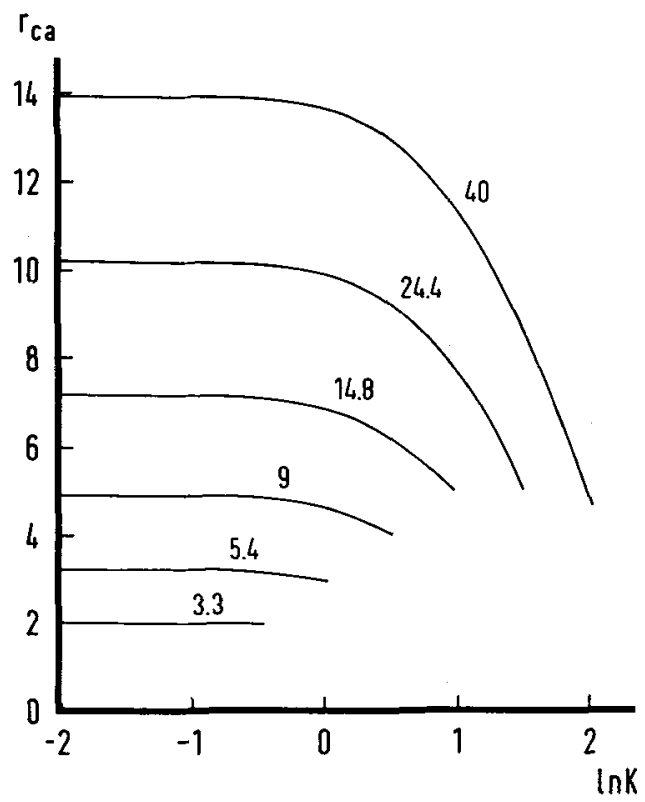

Fig. 2 - Dependence of $r_{c a}$ on Stokes number (K) for various values of $V_{m} / V_{0}$ (after R.P. Walker /8/).

entrapment at a fibre surface are normally referred to collectively as open gradient magnetic separation (OGMS) systems $/ 2 /$.

The basic mechanisms of magnetic particle entrapment and buildup on fibres in HGMS systems are now well understood as is the reduction in the performance of filters with increased particle loading $/ 2,6 /$. It is, therefore, sufficient to note here that, apart from various filter constants, the recovery of magnetic particles depends, in simple terms upon a single dimensionless variable $r_{c a}$. This quantity, a (normalised) particle capture cross-section per unit length of fibre (or wire, grid, etc.), can itself be expressed approximately as /2/

$$
r_{c a} \simeq \frac{3 \sqrt{3}}{4}\left(\frac{V_{m}}{V_{0}}\right)^{1 / 3} \text { for }\left|\frac{V_{m}}{V_{0}}\right| \gg 1,
$$

for paramagnetic particles in the longitudinal /2/ configuration. Here, $V_{O}$, is the average flow velocity of the particies, $V_{m}=2 \times B_{0} M_{s} b^{2} / 9 \mu_{o}$ na is the 'magnetic velocity' $16 /, b$ is the particle radius and $n$ is the fluid viscosity. It follows that, to a first approximation, $r_{c a}$ and, therefore, filter performance depends only on the ratio $V_{m} / V_{0}$. This is of considerable advantage in high-field HGMS systems where an increase in magnetic field to a higher value $\left(B_{0 S}\right)$ can be accompanied by a corresponding increase in $V_{o}$ (and, therefore, in production rate) without loss of performance. Some indication of this is provided in Fig. 1 where the recovery of iron oxide contamination from kaolin slurries /7/ is shown for various values of $B_{0}$ (ranging from 1.17 to $5.0 \mathrm{~T}$ ) and for corresponding values of $V_{0}$ adjusted to maintain a fixed value of $\alpha$ (i.e. of $\left.V_{m} / V_{0}\right)$. Apart from the low field data $(1.17 \mathrm{~T}$ ) the filter performance is seen to be in accordance with the above-described theory. There are, of course, other limits to the relationships expressed in Eq. (2). Fig. 2 shows computed values $/ 8 /$ of $r_{c a}$ as a function of $\log K$ where $K$ is the Stokes number. For values of $\log K>-0.18$, the simple relationship for filter performance embodied in Eq. (2) starts to break down. The Stokes number may be regarded as a measure of the balance between inertial and hydrodynamic forces and, at higher $K$ values, the former becomes increasingly 


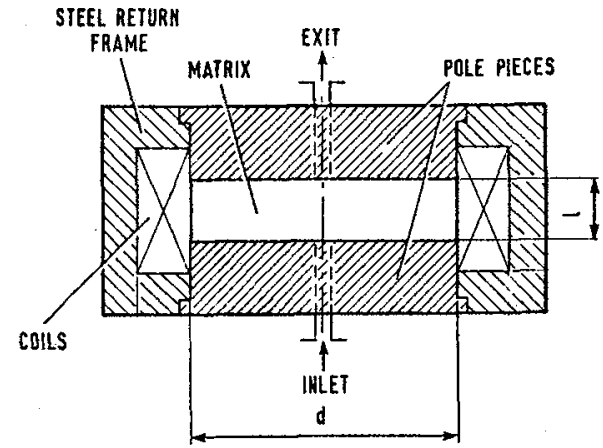

Fig. 3 - Conventional HGMS magnet system (after Gerber /5/). important. One consequence of this is that magnetic gas filtration which requires low packing density filters (and, correspondingly, high values of $r_{c a}$ ) is likely to benefit less from the use of very high fields.

\section{II - HIGH GRADIENT MAGNETIC SEPARATION (HGMS)}

In the light of the above analys is it may seem a little odd that, with their highfield advantage, $S / C$ magnets have, so far, made essentially no commercial impact upon HGMS. There are several reasons for this not the least of which is the effectiveness of the conventional HGMS cyclic system shown schematically in Fig. 3. Large-scale versions of this device, with values of $\mathrm{d}>2.0 \mathrm{~m}$ and with fields of up to $2.0 \mathrm{~T}$ have been produced widely on a commercial basis. The magnetic circuit and design of this device has been described in detail elsewhere $/ 5 /$ and only a brief mention is given here. The main advantages of this device include its robust construction (which is of considerable importance in the typically hostile environments found in minerals processing), its reliability (founded on conservative engineering principles) and the comparative ease with which the design may be scaled upwards in the dimension d of Fig. 3 . However, there are major disadvantages associated with these devices including comparatively high capital costs and operating power costs and an unfavourabiy long $(\sim 200 \mathrm{~s})$ 'dead' time (associated with magnetic field removal, followed by washing followed by field restoration). They are also liable to long-termmatrix contamination by ultra-fine particles.

The production rate, $P$, of a cyclic magnetic separator may be expressed $/ 6 /$ as a product of mass flux $\rho V_{0}\left(\pi d^{2}\right)$ and of the duty cycle $\left.n_{0} \tau / n_{0} \tau+n_{r} \tau+D\right)$ where $n_{0}$ is

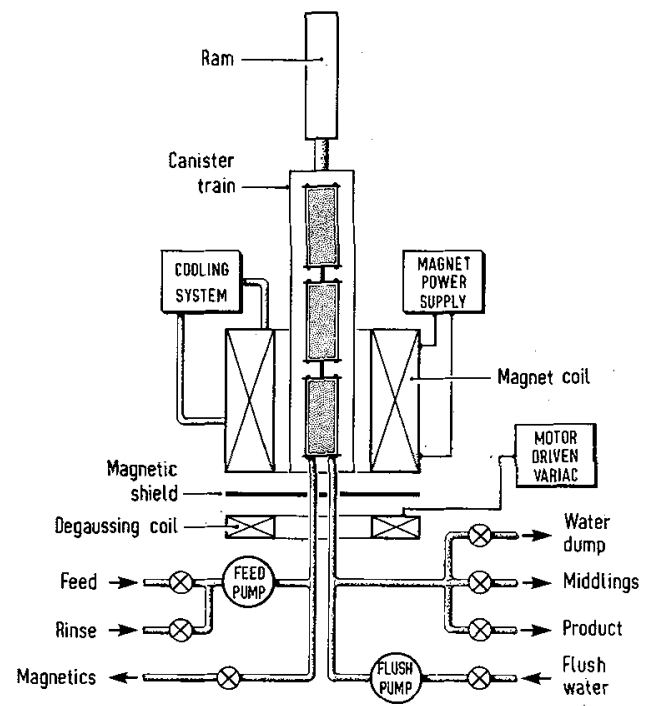

Fig. 4 - Schematic of cryogenic RC system (after Riley and Hocking /9/). the number of equivalent canisters of the feed stream processed before the recovery of the magnetic component becomes unacceptably low. When the flow is halted by appropriate heavy duty valve operation the matrix is rinsed with $n_{r}$ canisters of water (at speed $v_{0}$ and at full field) to remove essentially all of the non-magnetic fraction from the matrix. Typically $n_{r} \simeq 1$. Thus since $V_{0} \simeq \ell / \tau, P$ can be expressed in terms of the separating zone geometry as

$$
P=\rho A \ell n_{0} /\left[\left(n_{0}+n_{r}\right) \tau+D\right]
$$

$P$ then clearly depends largely on the value of $D$. For conventional (c) HGMS, $D_{c}=200 . s$ is not unreasonable $/ 6 /$. In large-scale $\mathrm{S} / \mathrm{C}$ magnets the corresponding value would be somewhere between $300 \mathrm{~s}$ and $600 \mathrm{~s}$. Under such circumstances all of the benefit of reduced residence time $\tau_{S}\left(=\tau_{c} / h\right)$ in $\mathrm{Eq}$. (3) from the magnetic field ratio $\mathrm{h}\left(=\mathrm{B}_{\mathrm{OS}} / \mathrm{B}_{\mathrm{OC}}\right)$ would be lost. This unfavourabie situation is retrieved in $S / C$ solenoids by the use of reciprocating canister (R.C.) systems. These were developed originally by scientists and engineers at English China Clays (ECLP) and based upon designs 


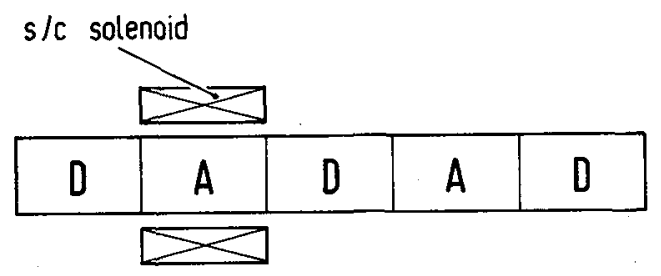

A: Active filter

D: Dummy filter

Fig. 5 -.R.C. train (after Watson et al $/ 10 /)$.

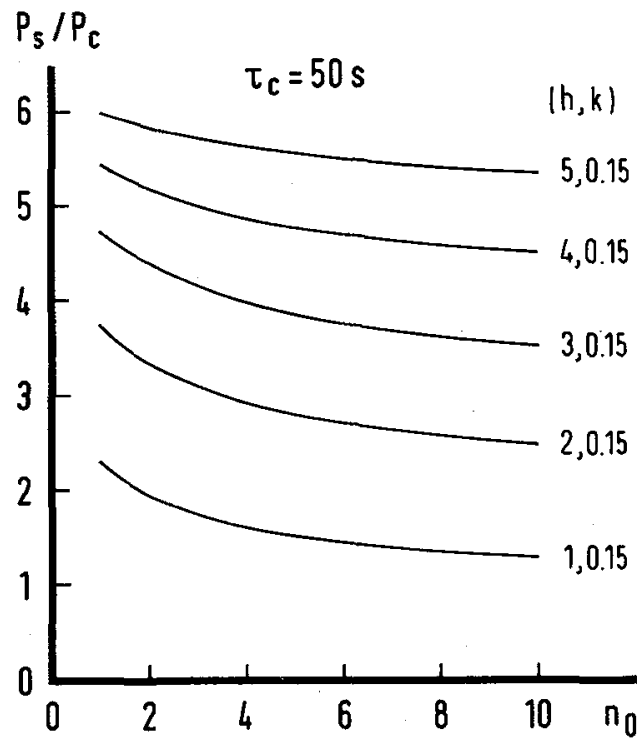

Fig. 6 - Production rate ratio for identical superconducting ( $\mathrm{s}$ ) and conventiona $\mathrm{l}$ (c) HGMS systems $\left(D_{c}=200 \mathrm{~s}, D_{\mathrm{s}}=30 \mathrm{~s}\right) / 6 \%$. ment of the refrigerator $\left(\sim 1 \mathrm{hh}^{-1}\right.$ for $300 \mathrm{~A}$ leads $) / 10 \%$. Second, and of greater imwith an estimated helium loss saving of order 3 to $4 \mathrm{lh}^{-1}$ in this type of system. These savings in helium consumption are also reflected in savings in capital costs of the liquefier and power costs of the refrigerator.

A number of advantages accrue from the R.C. system. As Fig. 4 indicates, the withdrawn canister may be shielded magnetically and de-gaussed during washing. The wash period itself can be increased to a value approaching the residence time $\left(n_{0} \tau_{C} / h=n_{0} \tau_{s}\right)$ of the second active canister in the $\mathrm{S} / \mathrm{C}$ magnet. Nevertheless, by far the greatest advantage of the R.C. system is in the reduction of $D$ to a value $\left(D_{S}\right)$ which is just the withdrawal (only) time of the loaded canister from the system. Watson /11/ has recently described a design specification of $10 \mathrm{~s}$ for a large bore $\left(\mathrm{d}_{1}=0.30 \mathrm{~m}\right) 5 \mathrm{~T}$ solenoid.

Fig. 6 shows production rate (P) comparisons between large-scale superconducting ( $s$ ) and conventional (c) HGMS systems of identical size as a function of $n_{0}$. Here, $\tau_{c}$ is fixed at $50 \mathrm{~s}$ and the value of the field ratio $h\left(=\mathrm{B}_{O S} / \mathrm{B}_{O C}\right)$ and the dead-time ratio 


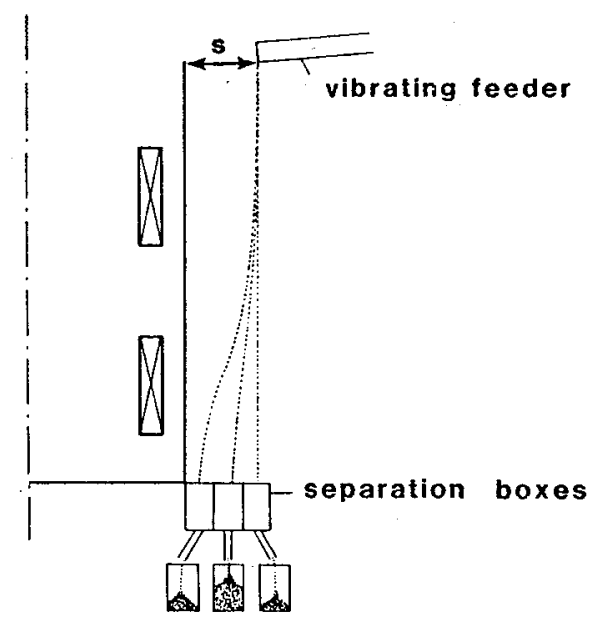

Fig. 7 - Schematic of S/C split-pair dry OGMS system. (Courtesy of H.K. Collan /3/)

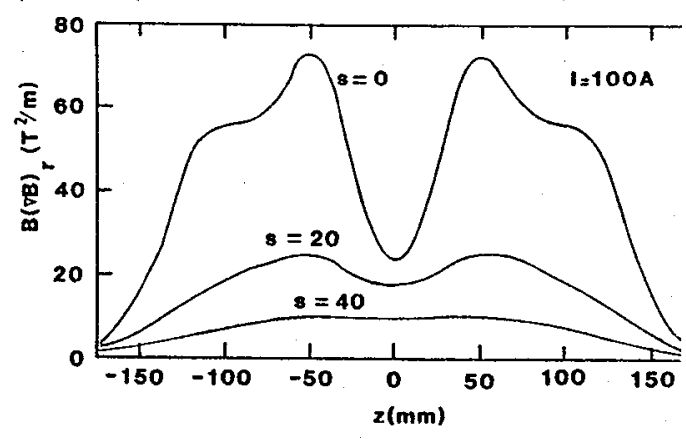

Fig. 8-Radial force density pattern (in units of $\mathrm{T}^{2} \mathrm{~m}^{-1}$ ). (Courtesy of H.K. Col1 an /3/)
$k\left(=D_{S} / D_{C}\right)$ are 1 isted in the set of curves. At low values of $n_{O}, P_{S} / P_{C}$ shows, in addition to the benefits of high fields a clear engineering advantage. When $D_{s}$ falls to $10 \mathrm{~s}$ this advantage becomes cons iderable. In addition to this production rate advantage, there are clear capital cost and operating cost advantages for the R.C. system. The former comes from the reduced physical scale of the R.C. system compared to its conventional counterpart. It has been estimated /11/ that a 5TR.C. system, with a production rate equivalent to a $2.1 \mathrm{~m}$ bore $2 \mathrm{~T}$ conventional system, will have a refrigeration power consumption of around $20 \mathrm{KW}$.

Clearly, problems do exist for R.C. cryogenic systems. Economic and technical reasons put an upper limit on the field ratio, $h$ of around $4 / 11 /$. Cryogenic solenoids operate comfortably up to about $8.0 \mathrm{~T}$ with conventiona $\mathrm{Nb}-\mathrm{Ti}$ wire. Above this field value costs escalate. In addition, in large-bore ( $\mathrm{d} \simeq 0.3 \mathrm{~m}$ ) $\mathrm{s} / \mathrm{C}$ solenoids, coil stresses become significant in terms of design and fabrication costs at fields greater than 5.0T. This is also an approximate upper field $1 \mathrm{imit}$ at which displacements of fine matrices in field gradients at the extremities of solenoids during canister withdrawal cease to be reversible /11/.

\section{III - OPEN GRADIENT MAGNETIC SEPARATION (OGMS)}

This separation method contrasts sharply with HGMS in its use of much smaller. force densities $\left(\sim 10^{8} \mathrm{Nm}^{-3}\right)$, generated by specially designed magnet coils, and of much larger force ranges (up to $0.1 \mathrm{~m}$ ) to deflect particle streams selectively and continuously towards collection points according to magnetic susceptibility. Such devices are also characterised by the absence both of a matrix and of a duty cycle relating to cyclic behaviour.

Early designs of 0GMS systems /12,13/ appear to have favoured the use of annular quadrupole magnets. Here the separating zone has a predominantly radial fieldgradient and force density pattern. One classical magnet system of this type used as long ago as 1968 by Kolm /2/ for the upgrading of molybdenum ores had the wet feed stream moving axially, in a pair of concentric pipes connected by perforation covering the surface of the inner pipe. Magnetic particles in the inner pipe diffuse radially outwards through the perforations under the influence of the radial field gradients. At the end of the magnet the magnetic and non-magnetic fractions exit separately. A number of papers were published by Cohen and Good /12/ on the use of a $S / C$ annular quadrupole for OGMS separation on dense mineral slurries. Here, the field and force density decrease sharply across the width of the pipe and separation of the mixed slurry is enhanced by promoting orbital motion of the slurry to allow all particles to enter the strongest part of the field. A slowly moving dense slurry of magnetic particles forms close to the cryostat wall and is discharged separately at a splitter at the exit point of the system. 
In more recent times S/C linear multipole OGMS systems appear to have completely replaced the annular systems for both wet and dry separation. The first reported system of this type was the (wet) solenoid pile separator described by Schonert et al /13/ in 1977 . More recently, three systems have been reported which reduce this 1 inear multipole to a S/C split pair driven in the cusp mode. The first of these is a pilotscale dry OGMS used successfully by Holman and Hise /14/ for the benefication of dry powdered coals. Here, the coal is fed under gravity (in a fashion not unlike that of Schonert et al) as an annular curtain adjacent to the inner surfaces of a vertical axis S/C split pair in a warm-bore cryostat of inner diameter $90 \mathrm{~mm}$. The force density at a radial distance of $30 \mathrm{~mm}$ from the coils is around $2.3 \times 10^{7} \mathrm{Nm}^{-3}$. The second two systems $/ 3,15 /$ prefer to carry out separation by allowing powdered mineral to fall under gravity as an annular curtain (Fig. 7) in close proximity to the cylindrical cryostat wall. The force density pattern of one of these $/ 3 /$ in units of $T^{2} m^{-1}$ as a function of the vertical distance from the horizontal plane of symmetry of the split pair is shown in Fig. 8 for various radial distances (s) from the cryostat wall with a peak value of about $5.0 \times 10^{7} \mathrm{Nm}^{-3}$ at $s=0$. An analysis of the force density patterns for $S / C$ split pair system of various sizes indicates that, unlike the solenoid systems of Figs. 3 and 4 , there is essentially no production rate advantage in scaling these systems upwards in size. Against that an OGMS system such as MASU $3 / 3 /$ can operate in the persistant mode with minimal heljum loss (using cryocoolers) and can process dry minerals at rates approaching $1 \mathrm{th}^{-1}$. It is probable that further development will indicate an optimum coil diameter enabling (with a warm bore) use of both the inner and outer surfaces of the coils.

\section{IV - LONG-TERM DEVELOPMENTS}

No review of this type would be complete without some predictions of future S/C separator development. By far the most promising of these is the cryogenic drum separator, a $S / C$ multipole device first conceived and patented by Ries et al/16/ in 1978. An industrial prototype of this device of approximate dimensions $1.0 \mathrm{~m}$ length and $1.0 \mathrm{~m}$ diameter has recently been under industrial test. Clearly, a device of this type combine the merits of OGMS with over 100 years of engineering experience in drum technology. Another potential development which combines the high-field advantages of magnetic flocculation with those of magneto-hydrostatic separation (MHS) is described in detail elsewhere in these Proceedings.

\section{REFERENCES}

/1/ KOLM H.H., OBERTEUFFER J.A. and KELLAND D.R., Sci. Am. 223 (1975) 46.

12/ BIRSS R.R. and PARKER M.R., Progress in Filtration and Separation, vol. 2 (Elsevier, 1981) 171.

13/ COLLAN H.K., KOKKALA M.A., MEINANDER T. and TOIKKA 0.E., Trans. Inst. Min. Meta11. (sect. C) 91 (1982) C5.

14/ OBERTEUFFER J.A., IEEE Trans. on Magn. MAG-10 (1974) 223.

15/ GERBER R., IEEE Trans. on Magn. MAG-18 (1982) 812.

16/ WATSON J.H.P., Proc. 6th Int. Cryogenic Eng. Conf., Grenoble (1976) 223.

17/ WATSON J.H.P. and HOCKING D., IEEE Trans. on Magn. MAG-11 (1975) 1588.

18/ WALKER R.P., MSC Dissertation, Univ. of Salford (1979).

19/ RILEY P.W. and HOCKING D., IEEE Trans. on Magn. MAG-17 (1981) 3299.

$110 /$ WATSON J.H.P., SCURLOCK R.G., SWALES A.W. and HOLDEN T.A., Proc. 9th Int. Eng. Conf. 6 Kobe (Butterworth 1982) 120.

/11/ WATSON J.H.P., Seminar, Univ. of Nijmegen, July 11 (1983).

112/ COHEN H.E. and GOOD J.A., IEEE Trans. on Magn. MAG-12 (1976) 552.

113/ SCHONERT K., SUPP A. and DORR H., 12th Int. MineraT Processing Congress, Meeting 4, paper no. 1, Sao Paulo, Brazil (1977).

14f HOLMAN A.S. and HISE E.C., Report no. ORNL-5764, Oak Ridge Nat Lab., May (1981).

115/ KOPP J. and GOOD J.A., IEEE Trans. on Magn. MAG-18 (1982) 833.

116/ RIES G., JUNGST K-P., FORSTER S., LEHMANN W. and UNKELBACH K-H., West German Patent No: 2650540 (1978). 